\title{
Irreversibilität der aeroben Spaltkraft bei optimaler Sauerstoffversorgung der Zelle
}

\begin{abstract}
Von F. Windisch, H. Haehn und W. Heumann
Aus dem Institut für Medizin und Biologie der Deutschen Akademie der Wissenschaften zu Berlin (Direktor: Prof. Dr. W. F ri e d ri c h), Abteilung Krebsforschung/Mikrobiologie
\end{abstract}

(Prof. Dr. F. W in dis c h)

(Z. Naturforschg. 8 b, 463-472 [1953]; eingegangen am 18. August 1953)

Herrn Professor Dr. Otto Warburg zum 70. Geburtstag

Mit der Entdeckung der Krebsglykolyse durch W a rbu rg sind zwei differente Arten von Spaltungsstoffwechsel bekannt geworden. Die aerob irreversible Spaltkraft ist nach W a r b u r g für alle malignen Zellen symptomatisch. Aber auch die Hefen und Milchsäurebakterien in der Natur verfügen, wie im folgenden nachgewiesen wird, über die Fähigkeit der aeroben Zuckerspaltung.

Die gärfähige Torulopsis, von $\mathrm{M}$ e y e rh of als Prototyp der aerob nicht-gärenden Hefen herausgestellt, bildet oxy- wie anoxybiotisch annähernd die gleichen Mengen Alkohol. Bei der zymagenen Umzüchtung von ursprünglich nur atmenden Torulazeen ist die Spaltkraft bereits in statu nascendi aerob irreversibel konstituiert. Die Meyerhofsche Korrelationstheorie von der aeroben Dominanz der Atmung über die Spaltung ist demnach in ihrem unitarischen Anspruch unsubstantiiert und zu reduzieren.

Die Hefezelle, die im ausgereiften, ruhenden Zustand keiner $\mathrm{O}_{2}$-Beeinflussung unterliegt, ist vegetativ $\mathrm{O}_{2}$-abhängig, sowohl in bezug auf die Proliferation selbst, als auch hinsichtlich der potentiellen Ausbildung der Stoffwechselkräfte. Im vegetativen Stadium tritt die P a s t e u r sche „conséquence de la vie sans air“ voll in Erscheinung. Für die fertig ausgebildete, nicht in Vermehrung befindliche Hefezelle gibt es indessen weder eine P a s t e u r sche Konsequenz der Anaerobiose, noch eine M e y e r h of sche Korrelation zwischen Oxy- und Anoxybiose.

$\mathrm{D}$ ie Gärung der Tumoren, die auf der Spaltung von Zucker zu Milchsäure beruht, ist 1923 von W a r b u r g ${ }^{1}$ entdeckt worden, zu einer Zeit, als man von den Gärungsfermenten, ja überhaupt von den Fermenten in chemischer Hinsicht noch nichts wußte. Seit der Entdeckung der Tumorgärung hat man sich immer wieder gefragt, ob wirklich alle Tumorzellen bei Sättigung mit Sauerstoff gären, und ob es wirklich keine normalen Körperzellen gibt, die bei optimaler Sauerstoffversorgung das gleiche tun.

Tatsächlich ist von der Gärung der Tumoren bis heute, so beantwortet W a rburg ${ }^{2} 1947$ die erste Frage, keine einzige Ausnahme gefunden worden, ob die Tumoren spontan gewachsen, ob sie künstlich erzeugt oder ob sie transplantiert waren; bei der Vielgestaltigkeit der Tumoren ein beachtenswertes Ergebnis. Zu der zweiten Frage bemerkt Warburg, daß Gewebe, die man aus dem Körper herausnimmt, im Verlauf des Absterbens ihr Atmungsvermögen schneller verlieren als ihr Gärungsvermö-

1 O. W arburg, Biochem. Z. 142, 317 [1923]; O. W a rburg, K. Pos ener u. E. Negelein, Biochem. Z. 152, 309 [1924]. gen, so daß es für alle aus dem Körper herausgenommenen Gewebe ein Zeitintervall gibt, in dem sie auch bei Sättigung mit Sauerstoff gären. Beschränkt man aber die zweite Frage, was man tun sollte, auf den Stoffwechsel der Körperzellen in situ, also auf die Ergebnisse der Methode der Gefäßpunktion, so ist bisher außer den Tumoren kein lebendes teilungsfähiges Gewebe gefunden worden, das im Körper bei Sättigung mit Sauerstoff gärt.

Nach Warburg gibt es also zwei Arten von Spaltungsstoffwechsel, je nach der Veranlagung der Zellen. Die eine, und zwar die der normalen Körperzellen in vivo, tritt als alternierende Zellfunktion nur unter anaeroben Bedingungen in Erscheinung, wird aber wieder vollkommen sistiert, sobald bei ausreichender Sauerstoffversorgung die Zellatmung von neuem einsetzt. Die aerob reversible Spaltkraft steht demnach in subordinierter Relation zur zellularen Oxydationskraft.

Im zweiten Falle dagegen - beim Krebsgewebe -

2 O. W a r burg, Ideen zur Fermentchemie der Tumoren, Abh. dtsch. Akad. Wiss. Berlin, math.-naturwiss. Kl. 1947. 
bedarf es nicht erst der anoxybiotischen Initialreaktion, der Pasteurschen „conséquence de la vie sans air", um den glykolytischen Spaltungsprozeß auszulösen. Die Krebsglykolyse verläuft unabhängig von dem umgebenden und in die Zelle eindiffundierenden Sauerstoff; sie kann durch $\mathrm{O}_{2}$-Einwirkung, selbst bei Sättigung mit reinem Sauerstoff, weder gehemmt noch eliminiert werden; sie ist gegenüber der zellularen Oxydationskraft autonom.

Beim Muskelgewebe ist nach Feststellungen Meyerhofs ${ }^{3}$ der anoxybiotisch initiierte Spaltungsstoffwechsel aerob reversibel; er wird wieder vollständig inhibiert, sobald bei erneutem $\mathrm{O}_{2}$-Zutritt eine adäquate $\mathrm{O}_{2}$-Konzentration im Reaktionsmedium erreicht ist (conséquence de la vie avec air). Die Konsequenz der Oxybiose, die sich beim Muskelgewebe sowie bei allen normalen tierischen Körperzellen in situ (W a r b u r g), bei Schimmelpilzen und Phanerogamen als Pasteursche Reaktion geltend macht, besteht nach Warburg für das Krebsgewebe nicht. Sie besteht aber auch nicht, entgegen der Annahme M e y e rh of s ${ }^{4}$, für Hefen und Milchsäurebakterien, was - in Verbindung mit früheren Ergebnissen ${ }^{5}$ - aus unseren nachfolgenden Untersuchungen in elementarer Weise hervorgeht.

\section{Experimenteller Teil}

Umstellung der Stoffwechselbilanz

Wir haben viele Mühe aufgewandt, um die Ursachen für die bestehenden Abweichungen zwischen den gasvolumetrischen und makrometrischen Stoffwechselbestimmungen bei Mikroorganismen ausfindig zu machen. Bei Einführung der Gasvolumetrie hatte Warburg ${ }^{6}$ bereits darauf hingewiesen, daß der Stoffwechsel von Bakterien „nur ausnahmsweise so einfach ist, daß er manometrisch bestimmt werden kann“. In jedem Falle empfiehlt Warburg, ,mit Hilfe der Gasanalyse festzustellen, daß andere Gase als Sauerstoff und Kohlensäure weder entstehen noch verschwinden“. Bei Hefen hat Wind is ch ${ }^{7}$ quantitativ ermittelt, daß sie „während der Gärung nicht unbeträchtliche Mengen flüchtiger Bestandteile“ zusammen mit der Kohlensäure abgeben. Indessen tre-

3 O. M e y e r h of, Asher Spiros Ergebn. d. Physiol. 22, 328 [1923].

4 O. M e y e rh of, Biochem. Z. 162, 43 [1925]; O. Meye rh of u. P. Finkle, Chem. d. Zelle Gewebe 12, 157 [1926].

5 F. Wind is ch, Ergebn. Enzymforsch. 2, 169 [1933].

6 O. W a r bu r g, Biochem. Z. 152, 51 [1924].

7 F. W in d is ch, Biochem. Z. 246, 332 [1932]. ten alle sonst noch vorzubringenden Einwände hinter unserem prinzipiellen Bedenken zurück, daß die experimentelle Stoffwechselbilanz, wie sie bisher im allgemeinen bei Mikroorganismen gebräuchlich ist, nicht die unbedingte Gewähr dafür bietet, daß die Stoffwechselgrößen Atmung und Gärung auch tatsächlich in jedem Falle einwandfrei und exakt erfaßt werden.

Mit der Messung des $\mathrm{O}_{2}$-Verbrauchs ist die wirkliche Atmungsgröße nur erfaßbar, wenn ideale Respiration $(R Q=1)$ in der Zelle vorherrscht. Sobald aber Oxydations- und Aufbauvorgänge nebeneinander ablaufen (z. B. erhöhter $\mathrm{O}_{2}$-Bedarf im vermehrungsfähigen Zustand der Organismen), sind keine Rückschlüsse vom $\mathrm{O}_{2}$-Verbrauch auf die Atmungsgröße möglich. Wird unter letzterem Umstand, wie üblich, die Gärungsgröße durch Differenzbestimmung festgelegt, indem von dem ermittelten Gesamt- $\mathrm{CO}_{2}$ eine dem $\mathrm{O}_{2}$-Verbrauch äquivalente $\mathrm{CO}_{2}$-Menge abgesetzt wird, so entsteht ein zweiter reziproker Fehler dadurch, daß die Gärung um den Betrag zu niedrig berechnet wird, der für die Atmung fälschlicherweise $\mathrm{zu}$ hoch eingesetzt wurde.

Bei der Nachprüfung der M e y e r h of schen Versuche mit Torulopsis * im W arburg-Apparat kamen wir auf den Gedanken, die hierbei entscheidenden Alkoholbestimmungen ${ }^{* *}$ in quantitativ exakter Weise dadurch möglich zu machen, daß wir jeweils eine größere Anzahl von Parallelansätzen im Warburg-Apparat ablaufen ließen und nach Versuchsbeendigung das Gärgut aus den einzelnen Küvetten verlustlos sammelten. Hierbei stellten wir fest, worauf später noch näher eingegangen wird,

* M e y e r h of (Biochem. Z. 162, 43, 62 [1925] führte hierzu aus: „Die Gärungsgröße der Torula in Stickstoff entspricht der größten von mir gemessenen Gärungsgröße der Kulturhefen, $Q_{\mathrm{CO}_{2}}=250$ bis 300 . Trotzdem gärt die Torula in Sauerstoff nicht. Die Torula verhält sich also genau so, wie sich nach unseren Versuchen der Kaltblüter- und Warmblütermuskel oder nach W a rbu r g das embryonale Gewebe hinsichtlich der Milchsäurespaltung des Zuckers verhält; die Sauerstoffatmung reich gerade aus, den in Stickstoff beträchtlich großen Spaltungsstoffwechsel rückgängig zu machen.“

${ }^{* *}$ M e y e r h of (l. c. ${ }^{*}$ S. 48) ging bei der Alkoholbestimmung, die er nach der Methode von Wid ma rk (Biochem. Z. 131, 473 [1922]) in modifizierter Form vornehmen ließ, stets nur von einer einzigen Küvette aus. Nach Abzentrifugieren der Hefe wurden 1 oder $2 \mathrm{ml}$ der klaren Zucker-Salz-Lösung (ohne vorherige Zerstörung des gebildeten Acetaldehyds und der aus der Hefe stammenden organischen Substanzen) in ein 5 bis $10 \mathrm{ml}$ fassendes Destillierkölbchen gefüllt; hieraus wurde der Alkohol abdestilliert, in Bichromatschwefelsäure aufgefangen und nach Zugabe von Jodkali mit n/100-Thiosulfat titriert. 
daß sich neben Acetaldehyd, der quantitativ erfaßbar ist, annähernd die gleichen Mengen Alkohol im aeroben wie im anaeroben Ansatz gebildet hatten.

Aus diesem für unsere weiteren Untersuchungen richtungweisenden Befund erkannten wir zugleich die grundlegende Bedeutung der Alkoholbestimmung für die Aufstellung einer präzisierteren und aufschlußreicheren Stoffwechselbilanz, als sie nach dem bisherigen Berechnungsmodus gezogen werden konnte. mikrobiellen Stoffwechselkräfte in ihrer spontanen Wirkungsfähigkeit zu erfassen, gingen wir von Zellen aus, die wir im Rahmen der vorgesehenen Messungen eigens für diesen Zweck aus der Natur isolierten.

$\mathrm{Da}$ die physiologische Beschaffenheit der Testorganismen für die richtige Auswertbarkeit der Stoffwechselversuche grundbestimmend ist, registrieren wir zunächst im folgenden die genaue Herkunft und Anzucht der in den weiter unten angeführten Versuchsbeispielen vorkommenden Hefen- und Bakterienarten: 2 Weinhefen (Sacch. ellipsoideus Hansen), Stamm W und Rie, beide aus spontan gärendem Traubenmost isoliert; 3 Torulopsisarten,

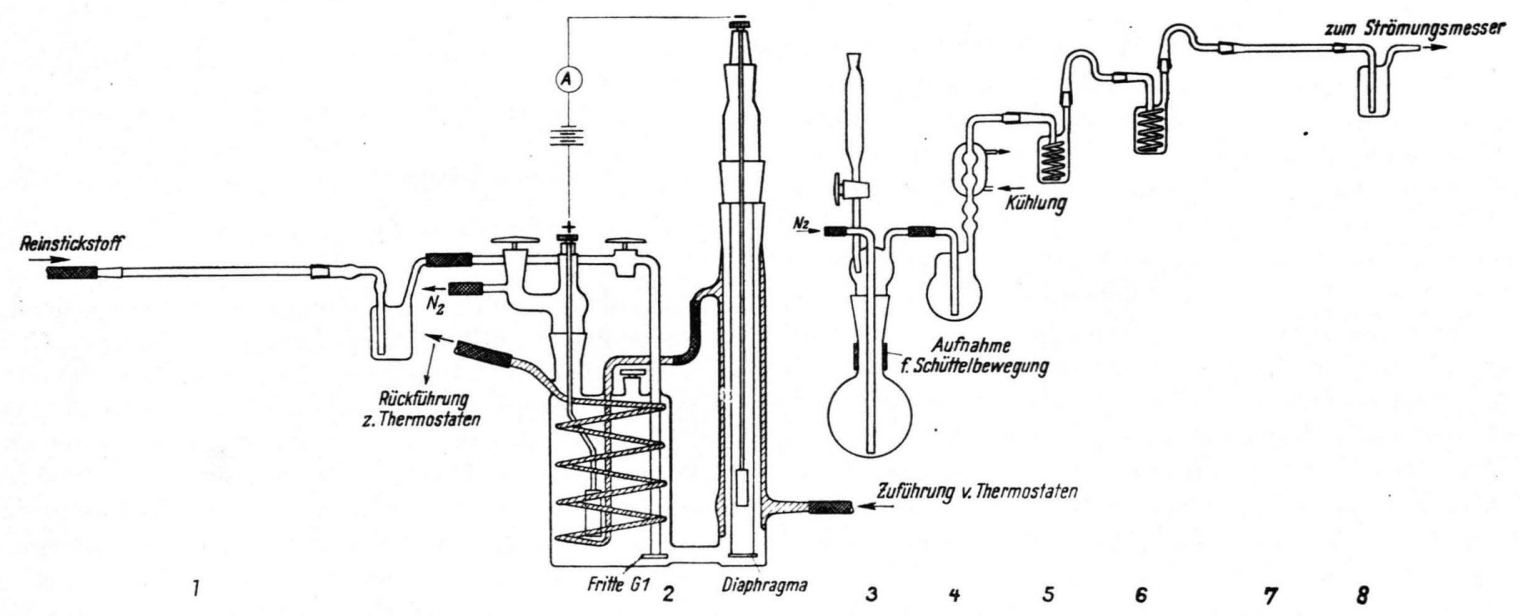

Abb. 1. Apparatur zur Makrometrie des Zellstoffwechsels: 1 Nachreinigung des Stickstoffs, 2 Elektrolyse- und Gasmischgefäß, 3 Reaktionsgefäß, 4 Chromschwefelsäure-Vorlage, 5 Schwefelsäure-Waschflasche, 6 Kaliapparat, 7 Kupfer-Quarzrohr zur Erfassung des Rest- $\mathrm{O}_{2}, 8$ Waschflasche mit Sperrflüssigkeit. Alternativ: Zwischen 2 und 3: Elektrochemisches $\mathrm{O}_{2}$-Meßgerät nach $\mathrm{T}$ ö d t ${ }^{*}$, wie in einer vorangehenden Arbeit ${ }^{* *}$ beschrieben. Anschließend an 3 : Gekühlte Vorlagen zum Ausfrieren übergehender Spuren von Alkohol.

Geht man künftig bei der Bilanzierung der Stoffwechselmessungen nicht von der dem $\mathrm{O}_{2}$-Verbrauch äquivalenten „Atmungskohlensäure“, sondern von der effektiven Gärungskohlensäure aus, die in Äquivalenz zum Gärungsalkohol eine festumrissene Größe darstellt, und bringt letztere von der Gesamt- $\mathrm{CO}_{2}$ in Abzug, so entspricht die mit dem restierenden $\mathrm{CO}_{2}$ Betrag korrespondierende $\mathrm{O}_{2}$-Menge der tatsächlichen Atmungsgröße, während gleichzeitig hierbei der überschüssige Sauerstoff, den die Zelle evtl. für andere als für respiratorische Zwecke beansprucht, in quantitativer Weise erfaßt werden kann.

\section{Das verwendete Zellmaterial}

Die Kulturorganismen gelten wegen ihrer spezialisierten technischen Hochzüchtung als atypisch 8 . Um die

8 O. M e y e r h of, Biochem. Z. 162, 43, 62 [1925]; H. H a e h n, Biochemie der Gärungen, Berlin 1952, S. 270.

9 F. H a y d k u. H. H a e hn, Biochem. Z. 128, 567 [1922].
Stamm $\mathrm{Hb}, \mathrm{Bi}$ und $\mathrm{M}$, ersterer von Holunderbeeren, der zweite aus Birkensaft und letzterer von Honigwaben isoliert, alle 3 Stämme ursprünglich nicht-gärend; 2 Vertreter von Milchsäurebakterien, und zwar Bact. lactis acidi Leichmann, aus spontan gesäuerter Milch (Luftinfektion) isoliert, und Bact. Delbrücki Leichmann, aus einer bei $48^{\circ}$ gehaltenen Roggenmalzmaische (Rohfruchtinfektion) gewonnen. Die zum Vergleich der Gärungsintensitäten einbezogene untergärige Kulturhefe, Stamm F, war unserer Organismensammlung entnommen.

Die Weinhefen wurden in gefiltertem, sterilisiertem Traubenmost hergeführt. Die zymagene Hochzüchtung der Torulopsisarten, also ihre Umwandlung von nichtgärenden in kräftig gärende Hefen, erfolgte nach der von $\mathrm{Hayduck}$ und $\mathrm{Haehn}{ }^{9}$ ausgearbeiteten Vorschrift. Bact. Delbrücki Leichmann wurde in sterilisierter Roggen- und Gerstenmalzmaische unter Kreidezusatz bei $42^{\circ}$ im Thermostaten zur Massenzüchtung angesetzt. Die Ernte wurde nach 48-stdg. Kultivierungszeit mittels frak-

* Gemeinschaftsarbeit von F. Tö dt u. G. T e s k e sowie von F. Wind is ch, W. He umann u. Chr. G o s li c h, Bio. Z. 323, 192 [1952].

${ }^{* *}$ F. Windis ch u. W. He u m a n n, Naturwiss. 39, 329 [1952]. 
tionierter Separierung bewerkstelligt, wobei es uns gelang, die Bakterien von den schwereren Maische- und Kreidepartikeln mit befriedigender Ausbeute zu trennen. Die Massenzüchtung von Bact. lactis acidi Leichmann wurde auf Würzeagar vorgenommen. Die gut bewachsenen Platten schwemmten wir mit steriler Kochsalzlösung (0,85-proz.) ab und ernteten darauf die Bakterien, nach dreimaliger Waschung auf der Zentrifuge, mit guter Ausbeute. Die untergärige Kulturhefe wurde in 12-proz. Würze angezüchtet.

\section{Methodisches}

In den Reihenversuchen kamen je $100 \mathrm{ml}$ Würze $\left.p_{\mathrm{H}} 5,50-5,60\right)$ von 10 bzw. $12 \%$ Extraktgehalt zum biologischen Ansatz. Die Aussaatmenge wurde, nach vorheriger Feststellung, jeweils so hoch bemessen, daß keine Zellvermehrung - auf Grund der natürlichen Volumbegrenzung des Wachstums 10 - eintreten konnte. In diesem Zusammenhang sei noch einmal darauf hingewiesen, daß zwischen dem Stoffwechsel der fertig ausgebildeten, ruhenden Zelle (Erhaltungsstoffwechsel) und dem der sprossenden, sich vermehrenden grundsätzlich unterschieden werden muß, um widersprechende Interpretationen der biologischen Ergebnisse zu vermeiden. Allein schon bei vermehrungsfähiger Tendenz, wenn überhaupt noch kein sichtbarer Zellzuwachs feststellbar ist, ändert sich das Stoffwechselbild gegenüber dem Ruhezustand der Zelle 11. Außer der Trockensubstanz der Organismen wurde auch ihre Zellenzahl $/ \mathrm{mm}^{3}$ bestimmt, so daß stets ein Rückschluß auf die Stoffwechselleistung der einzelnen Zelle gezogen werden kann.

Die Mikroansätze im Warburg-Apparat waren genauestens auf die von $\mathrm{M}$ eyerhof 12 getroffenen Anordnungen abgestellt, da es in vorliegender Arbeit als Ausgangspunkt der Problematik entscheidend darauf ankam, einzelne der M e y e r h of schen mikrobiologischen Stoffwechselversuche in exakter Weise zu reproduzieren.

Die makrometrischen Stoffwechselbestimmungen (M.St.Bestimmungen), deren apparative Grundlage aus Abb. 1 $\mathrm{zu}$ ersehen ist, werden unter Zuleitung von synthetischer Luft (nicht von Sauerstoff) bei $28^{\circ}$ (Thermoregulation) durchgeführt. Die Berechnungen beziehen sich auf $1 \mathrm{~g}$ Organismen-Trockensubstanz in $200 \mathrm{ml}$ Substrat (Zuckerphosphatlösung) je Stde. Versuchsdauer. Der Alkohol ist in $\mathrm{mg}$, die Gaswerte $\left(\mathrm{O}_{2}\right.$ und $\left.\mathrm{CO}_{\vartheta}\right)$ sind in $\mathrm{ml}$ angegeben. Es kann, sofern die Zellenzahl $/ \mathrm{mm}^{3}$ ermittelt ist, auf die Leistung der einzelnen Zelle umgerechnet werden. In Anlehnung an die von $\mathrm{W}$ a r bu r g eingeführte Nomenklatur der Stoffwechselquotienten haben wir folgende abgekürzte Bezeichnungen für die einzelnen $Q$-Werte gewählt:

10 W. S c h m i d, Z. Naturforschg. 7 b, 217 [1952].

11 O. Warburg, Pflügers Arch. ges. Physiol. Menschen Tiere 158, 189 [1914].

12 O. M e y e r h of, Biochem. Z. 162, 43, 54/55, 59/62 [1925].

13 E. W e in ig, Dtsch. Z. ges. gerichtl. Med. 40, 318 [1951].

$$
\begin{aligned}
& Q_{\mathrm{Al}}^{\mathrm{N}_{2}}=\text { anaerobe Alkoholbildung (best.), } \\
& Q_{\mathrm{Al}}^{\mathrm{O}_{2}} \quad=\text { aerobe Alkoholbildung (best.), } \\
& Q_{\mathrm{CO}_{2}}^{\mathrm{N}_{2}}=\text { anaerobe Gärungskohlensäure (best.), } \\
& Q_{\mathrm{CO}_{2}}^{\mathrm{O}_{2}}=\text { aerobe Gesamtkohlensäure (best.), } \\
& G-Q_{\mathrm{CO}_{2}}^{\mathrm{O}_{2}}=\text { aerobe Gärungskohlensäure } \\
& \text { (ber. als Äquivalent von } Q_{\mathrm{Al}}^{\mathrm{O}_{2}} \text { ), } \\
& Q_{\mathrm{O}_{2}} \quad=\text { gesamter } \mathrm{O}_{2} \text {-Verbrauch (best.), } \\
& A-Q_{\mathrm{O}_{2}}=\text { Atmungs- } \mathrm{O}_{2} \text {-Verbrauch (ber. als Äquivalent } \\
& \text { aus der Differenz von } Q_{\mathrm{CO}_{2}}^{\mathrm{O}_{2}} \text { minus } \mathrm{G}-Q_{\mathrm{CO}_{2}}^{\mathrm{O}_{2}} \text { ), } \\
& \ddot{U}-Q_{\mathrm{O}_{3}}=\text { überschüssiger } \mathrm{O}_{2} \text {-Verbrauch (ber. aus der } \\
& \text { Differenz von } \left.Q_{\mathrm{O}_{2}} \text { minus } A-Q_{\mathrm{O}_{2}}\right) \text {. }
\end{aligned}
$$

Der Äthylalkohol wurde, sofern er in ausreichender Menge anfiel, quantitativ durch Ermittlung des spez. Gew. nach anreichernder Destillation bestimmt. Bei kleineren Mengen von Äthylalkohol wurde die quantitative Analyse nach der von Weinig ${ }^{13}$ modifizierten Widmark Methode ausgeführt, deren Zuverlässigkeit vorher von Herm Dr. R o the $\mathrm{nbach}$ in unserem Laboratorium durch vergleichende Parallelbestimmungen mit der spezifischen Dinitrobenzoylchlorid-Methode nach $\mathrm{K} \mathrm{l} \mathrm{u} \mathrm{g} \mathrm{e}{ }^{14}$ überprüft worden war. Auch die Darstellung in substantia als $p$-Nitrobenzoesäure-äthylester (Schmp. 57 ${ }^{\circ}$ ) nach der Vorschrift von Buchner und $\mathrm{Me}$ is enheimer ${ }^{\mathbf{1 5}}$ diente uns anfänglich zur Identifizierung des Äthylalkohols. - Die Milchsäure wurde quantitativ nach $\mathrm{F}$ ü $\mathrm{rth}$ und $\mathrm{Charnass}$ in der Modifikation von $\mathrm{Friede-}$ $m$ an $n$ und $\mathrm{Gr}$ a e s e 16 bestimmt, nachdem das Substrat zuvor nach $\mathrm{S} \mathrm{c}$ h e $\mathrm{n} \mathrm{k}$ enteiweißt und die Milchsäure mit Amylalkohol extrahiert worden war. Sie wurde über $\left(\mathrm{C}_{3} \mathrm{H}_{5} \mathrm{O}_{3}\right)_{2} \mathrm{Zn} \cdot 3 \mathrm{H}_{2} \mathrm{O}$ als $\mathrm{ZnO}$ identifiziert.

\section{Stoffwechselversuche}

Weder Pasteur ${ }^{17}$ noch $\mathrm{Meyerhof}{ }^{18}$ ist es unter normalen physiologischen Bedingungen gelungen, den Gärungsstoffwechsel der Kulturhefen in nennenswertem Maße durch $\mathrm{O}_{2}$-Einwirkung auf Atmung umzustimmen. Vergeblich versuchte $\mathrm{Pa}$ steur, seine These von der Gärung als „conséquence de la vie sans air“ an den typischen Gärungserregern, den Kulturhefen, zu beweisen. Die Resistenz ihrer Gärkraft unter aeroben Verhältnissen

14 H. K l u g e, Z. Unters. Lebensmittel 78, 449 [1939].

15 E. B u chner u. J. M e is e $n$ hei m e r, Ber. dtsch. chem. Ges. 38, 625 [1905].

16 T. E. F ri e d e m a n n u. J. B. Gra e se r, J. biol. Chemistry 100, 291 [1933].

17 L. P a s t e u r, Etudes sur la bière, Paris 1876.

18 O. M e y e r h of, Biochem. Z. 162, 43, 62 ff. [1925]. 


\begin{tabular}{|c|c|c|c|c|c|c|c|c|}
\hline \multicolumn{5}{|c|}{ Weinhefe } & \multicolumn{4}{|c|}{ Weinhefe } \\
\hline \multicolumn{5}{|c|}{$\begin{array}{l}\text { Sacch. ellipsoideus Hansen } \\
\text { Stamm W }\end{array}$} & \multicolumn{4}{|c|}{$\begin{array}{l}\text { Sacch. ellipsoideus Hansen } \\
\text { Stamm Rie }\end{array}$} \\
\hline \multirow{3}{*}{ Stdn. } & \multicolumn{8}{|c|}{ Bezogen auf $100 \mathrm{ml}$ Gärsubstrat } \\
\hline & \multicolumn{2}{|c|}{ anaerob } & \multicolumn{2}{|c|}{ aerob } & \multicolumn{2}{|c|}{ anaerob } & \multicolumn{2}{|c|}{ aerob } \\
\hline & $\begin{array}{c}\text { Alkohol } \\
\text { in } \\
\text { Gew.- } \%\end{array}$ & $\begin{array}{c}\mathrm{CO}_{2-} \\
\text { Menge } \\
\text { in } \mathrm{g}\end{array}$ & $\begin{array}{l}\text { Alkohol } \\
\text { in } \\
\text { Gew.- } \%\end{array}$ & $\begin{array}{l}\mathrm{CO}_{2-} \\
\text { Menge } \\
\text { in } \mathrm{g}\end{array}$ & $\begin{array}{c}\text { Alkohol } \\
\text { in } \\
\text { Gew.- } \%\end{array}$ & $\begin{array}{c}\mathrm{CO}_{2-} \\
\text { Menge } \\
\text { in } \mathrm{g}\end{array}$ & $\begin{array}{l}\text { Alkohol } \\
\text { in } \\
\text { Gew.- } \%\end{array}$ & $\begin{array}{l}\mathrm{CO}_{\varepsilon-} \\
\text { Menge } \\
\text { in } \mathrm{g}\end{array}$ \\
\hline $\begin{array}{r}24 \\
48 \\
72 \\
96 \\
120\end{array}$ & $\begin{array}{l}0,56 \\
1,49 \\
2,48 \\
2,69 \\
2,87\end{array}$ & $\begin{array}{l}0,52 \\
1,43 \\
2,41 \\
2,58 \\
2,77\end{array}$ & $\begin{array}{l}0,48 \\
1,33 \\
2,52 \\
2,75 \\
2,79\end{array}$ & $\begin{array}{l}0,47 \\
1,32 \\
2,50 \\
2,74 \\
2,83\end{array}$ & $\begin{array}{l}0,76 \\
1,78 \\
2,69 \\
2,84 \\
2,96\end{array}$ & $\begin{array}{l}0,73 \\
1,71 \\
2,57 \\
2,75 \\
2,85\end{array}$ & $\begin{array}{l}0,82 \\
1,60 \\
2,81 \\
2,98 \\
3,05\end{array}$ & $\begin{array}{l}0,82 \\
1,58 \\
2,78 \\
3,02 \\
3,08\end{array}$ \\
\hline
\end{tabular}

Tab. 1. Aanaerobe und aerobe Alkoholbildung sowie $\mathbf{C O}_{2}$-Entwicklung der Weinhefen (Sacch. ellipsoideus Hansen) Stamm W und Rie in 12-proz. Würze bei nicht-zuwachsfähiger Aussaatmenge. Raumtemperatur $5^{\circ}$.

führte M e y e r h of darauf zurück, daß ihr Atmungsvermögen infolge fortgesetzter technischer Hochzüchtung auf Gärung eine weitgehende Einbuße erlitten habe; zugleich bekräftigte er damit seinen Standpunkt, daß bei den wilden Hefen dasselbe Stoffwechselprinzip der aeroben Umschaltung von Gärung auf Atmung vorherrsche wie beim Kaltblüter- und Warmblütermuskel. Am Beispiel von Torulopsis utilis vermeinte er, die Richtigkeit seiner Theorie bewiesen zu haben.

Nichts lag nun näher, als - im Bemühen um die Klarstellung dieses wichtigen biologischen Fragenkomplexes - von Hefen und Bakterien auszugehen, die in der Natur, umgeben von atmosphärischen $\mathrm{O}_{2^{-}}$ Einflüssen, aufgewachsen sind und die spezifische Eigenschaft besitzen, in zuckerhaltigen Säften und Lösungen eine spontane Gärung hervorzurufen, wie es z. B. von der Weingärung oder natürlichen Milchsäuerung her allgemein bekannt ist.

\section{Versuche mit Weinhefen}

Bei der unbegrenzten Zahl von Weinhefen (Spezies Sacch. ellipsoideus Hansen), die aus der Natur in Weingärten und Weinkellereien unter wechselnden Lebensbedingungen anfallen, konnten wir ein umfangreiches statistisches Untersuchungsmaterial zusammentragen. An den nachfolgenden beiden Beispielen zeigen wir ihr Verhalten gegenüber intensiver Sauerstoff-Einwirkung (bei $\mathrm{O}_{2}$-Sättigung des Mediums).

Ansatz 1: 10 Gärkolben mit je $100 \mathrm{ml} 12$-proz. Würze $\left(p_{\mathrm{H}} 5,56\right)$; je Charge 1,4 g abgepreßte Weinhefe Stamm W (nicht-zuwachsfähige Aussaatmenge); Wassergehalt der Hefe: $77,4 \%$, Zellenzahl $/ \mathrm{mm}^{3}$ : 67200 ; anaerob: bei schwachem $\mathrm{N}_{2}$-Gasstrom (etwa 20 Gasblasen/min), aerob: bei schwachem $\mathrm{O}_{2}$-Gasstrom (etwa $20 \mathrm{Gasblasen} / \mathrm{min}$ ). Raumtemperatur $5^{\circ}$.

M.St.-Bestimmungen:

$$
\begin{aligned}
& Q_{\mathrm{Al}}^{\mathrm{N}_{2}}=960 ; \quad Q_{\mathrm{Al}}^{\mathrm{O}_{2}}=973 ; \\
& Q_{\mathrm{CO}_{2}}^{\mathrm{N}^{2}}=465 ; \quad Q_{\mathrm{CO}_{2}}^{\mathrm{O}_{2}}=483 ; \quad G-Q_{\mathrm{CO}_{2}}^{\mathrm{O}_{2}}=471 ; \\
& Q_{\mathrm{O}_{2}}=-12 ; \quad A-Q_{\mathrm{O}_{2}}=-12 ; \quad \ddot{U}-Q_{\mathrm{O}_{2}}=0 .
\end{aligned}
$$

Ansatz 2: 10 Gärkolben mit je $100 \mathrm{ml}$ 12-proz.-Würze $\left(p_{\mathrm{H}} 5,56\right)$; je Charge 1,4 g abgepreßte Weinhefe Stamm Rie (nicht-zuwachsfähige Aussaatmenge); Wassergehalt d. Hefe: $78,6 \%$, Zellenzahl $/ \mathrm{mm}^{3}: 77600$; weitere Arbeitsbrdingungen wie bei Ansatz 1. Raumtemperatur $5^{\circ}$.

M.St.-Bestimmungen: Im Bereich der bei Weinhefe W angegebenen M.St.-Werte liegend.

In der nachstehenden Tab. 1 sind die von $24 \mathrm{zu}$ 24 Stdn. in 12-proz. Würze ermittelten Zahlenwerte der aeroben bzw. anaeroben Alkoholbildung und $\mathrm{CO}_{2}$-Entwicklung innerhalb von 5 Gärtagen zusammengestellt.

Die Gegenüberstellung der aeroben bzw. anaeroben Alkoholbildung und $\mathrm{CO}_{2}$-Entwicklung in Tab. 1 gibt eindeutig zu erkennen, daß bei den Weinhefen (von der Spezies Sacch. ellipsoideus Hansen) trotz maximaler Sauerstoffversorgung kein hervortretender Unterschied im Vergleich zu den anaeroben Gäransätzen besteht. Dieses Ergebnis hat sich auch durchgehend bei allen sonstigen mit Weinhefen angestellten Analogieversuchen bestätigt. Die wilden, aus der Natur stammenden Weinhefen sind also in ihrer Gärkraft gegenüber der $\mathrm{O}_{2}$-Einwirkung genau 
so resistent wie die Kulturhefen, d. h. ihre Spaltkraft ist aerob irreversibel.

\section{Versuche mit Torulopsis}

Die Torulopsishefe beansprucht in der Zellstoffwechsellehre insofern eine besondere Beachtung, als Meyerh of ${ }^{19}$ an ihr nachgewiesen zu haben vermeinte, daß auch die Gärhefen, mit Ausnahme der „kulturell entarteten“"Betriebshefen, dem Gesetz der aeroben Umschaltung von Spaltung auf Atmung unterworfen seien, woraus er die weittragende Folgerung ableitete, daß das Korrelationsprinzip des Muskels - Sistierung des Spaltstoffwechsels in der Oxybiose nebst Resynthese des Stabilisierungsproduktes - in der gesamten belebten Natur vorherrsche.

\begin{tabular}{|c|c|c|c|c|c|}
\hline Hefeart & $\begin{array}{c}\text { Hefe- } \\
\text { menge } \\
\text { i. Trock. } \\
\text { Subst. } \\
\text { mg }\end{array}$ & $\begin{array}{c}\text { Konzentr. d. } \\
\text { Zucker- } \\
\text { phosphat- } \\
\text { lösung } \\
\%\end{array}$ & $\begin{array}{c}\text { Sub- } \\
\text { strat- } \\
\text { menge } \\
\mathrm{ml}\end{array}$ & $\begin{array}{c}\text { Ver- } \\
\text { suchs- } \\
\text { dauer } \\
\text { min }\end{array}$ & $\begin{array}{c}\text { Me- } \\
\text { thode }\end{array}$ \\
\hline $\begin{array}{c}\text { Toru- } \\
\text { lopsis- } \\
\text { Stamm } \\
\text { M }\end{array}$ & 2,2 & 2,5 & 3,0 & $60^{*}$ & $\begin{array}{c}\text { Käst- } \\
\text { chen- } \\
\text { me- } \\
\text { thode }\end{array}$ \\
\hline
\end{tabular}

* Bei $30^{\prime}$ und $40^{\prime}$ treten die gleichen Reaktionen ein.

Tab. 2. Schema eines Einzelansatzes.

Bei Wiederholung der entscheidenden Meyerh of schen Versuche mit Torulopsis im Warburg Apparat gingen wir jedesmal von 22 Einzelansätzen in Küvetten aus und hielten uns dabei streng an die von Meyerhof gewählten Versuchsbedingungen.

\begin{tabular}{|c|c|c|c|c|c|c|c|}
\hline \multirow{3}{*}{$\begin{array}{l}\text { Versuchs- } \\
\text { Auswertung }\end{array}$} & \multirow{3}{*}{$\begin{array}{c}\begin{array}{c}\text { Hefemenge } \\
\text { in Trock.-Subst. }\end{array} \\
\mathrm{mg}\end{array}$} & \multicolumn{2}{|c|}{$\begin{array}{l}\text { Gesamte Alkoholbildung } \\
\text { in } 66 \mathrm{mg} \text { Gärlösung }\end{array}$} & \multicolumn{2}{|c|}{$\begin{array}{l}\text { In } 1 \text { Stde. von } 1 \mathrm{mg} \\
\text { Trock.-Hefe gebildete } \\
\text { Alkoholmenge }\end{array}$} & \multicolumn{2}{|c|}{$\begin{array}{l}\text { Aus dem Alkohol, je Stde } \\
\text { und mg Trock.-Hefe, be- } \\
\text { rechnete } \mathrm{CO}_{2} \text {-Menge }\end{array}$} \\
\hline & & \multicolumn{2}{|c|}{$\mathrm{mg}$} & \multicolumn{2}{|c|}{$\mathrm{mg}$} & \multicolumn{2}{|c|}{$\mathrm{mm}^{3}$} \\
\hline & & anaerob & aerob & anaerob & aerob & anaerob & aerob \\
\hline Maxir & \multirow{3}{*}{48,4} & 22,6 & 23,2 & 0,47 & 0,48 & 227 & 233 \\
\hline Minimaly & & 21,8 & 22,4 & 0,45 & 0,46 & 217 & 225 \\
\hline Mittelwert & & 22,2 & 22,8 & 0,46 & 0,47 & 222 & 229 \\
\hline
\end{tabular}

Tab. 3. Maximale, minimale und mittlere Alkoholwerte nebst der dazugehörigen berechneten $\mathrm{CO}_{2}$-Menge

Aber noch in anderer Hinsicht sind die Torulazeen von biologischem Interesse, weil sie, im Gegensatz z. B. zu den wilden Weinhefen, von Natur aus meistens nicht gärfähig sind, sondern ausschließlich atmen. Infolge ihrer Gärunfähigkeit steht es außer Zweifel, daß sie nicht irgendwie durch anaerobe Einflüsse vorbelastet sind. Sollen sie in gärende Zellen umgewandelt werden, so gelingt das, entgegen der strengen Auslegung der Pasteurschen „conséquence de la vie sans air", nicht bei völliger Abwesenheit von Sauerstoff, sondern nur unter vermindertem $\mathrm{O}_{2^{-}}$ Partialdruck (Lufthungerzüchtung), und zwar aus dem einfachen Grunde, weil die nicht-gärfähigen Torulazeen außerstande sind, sich anoxybiotisch fortzupflanzen ${ }^{20}$. Die gerade erst im zymagenen Umzüchtungsprozeß ausgebildete Spaltfähigkeit ist nach unseren Feststellungen ${ }^{21}$ - abweichend von denen M e y e r h of s - sozusagen in statu nascendi aerob irreversibel konstituiert, worauf im nachfolgenden noch zurückgekommen wird.

19 O. M e y e r h of , Biochem. Z. 162, 43, 59 ff. [1925].

$20 \mathrm{~F}$. Wind is ch, W. He umann u. Chr. Gos li c h, Z. Naturforschg. 8 b, 305 [1953].
In Tab. 2 ist das Schema eines derartigen Einzelansatzes angeführt.

Nach Versuchsbeendigung töteten wir die Hefe ab und vereinigten in quantitativer Weise die 22 Einzelansätze, welche insgesamt $66 \mathrm{ml}$ Substrat und $48,4 \mathrm{mg}$ Hefe in Tr.S enthielten. Darauf ermittelten wir in der angesammelten Gärflüssigkeit den Alkoholgehalt. Aus einer Versuchsreihe mit Torulopsis, Stamm M, sind in Tab. 3 die maximal und minimal gefundenen bzw. berechneten mittleren Alkoholwerte nebst den dazugehörigen $\mathrm{CO}_{2}$-Mengen (ber.) zusammengestellt.

Aus dem obigen Beispiel (Tab. 3) geht hervor, daß Torulopsis, Stamm M, welche gerade erst im Verlauf ihrer zymagenen Umzüchtung zuckerspaltende Befähigung erlangt hat, diese Eigenschaft auch unter der Einwirkung von Sauerstoff beibehält, wie ihre aerobe Alkoholgärung bezeugt, welche der anaeroben gleichwertig ist.

Anschließend gingen wir dazu über, die aus der Warburg-Apparatur zum Zwecke der Analysierung vereinigten Mikrogärungen nunmehr sogleich in

21 Gemeinschaftsarbeit von F. W ind is ch u. D.S ti er a n d sowie von $\mathrm{H}$. $\mathrm{H}$ a e h n, Protoplasma (im Druck). 


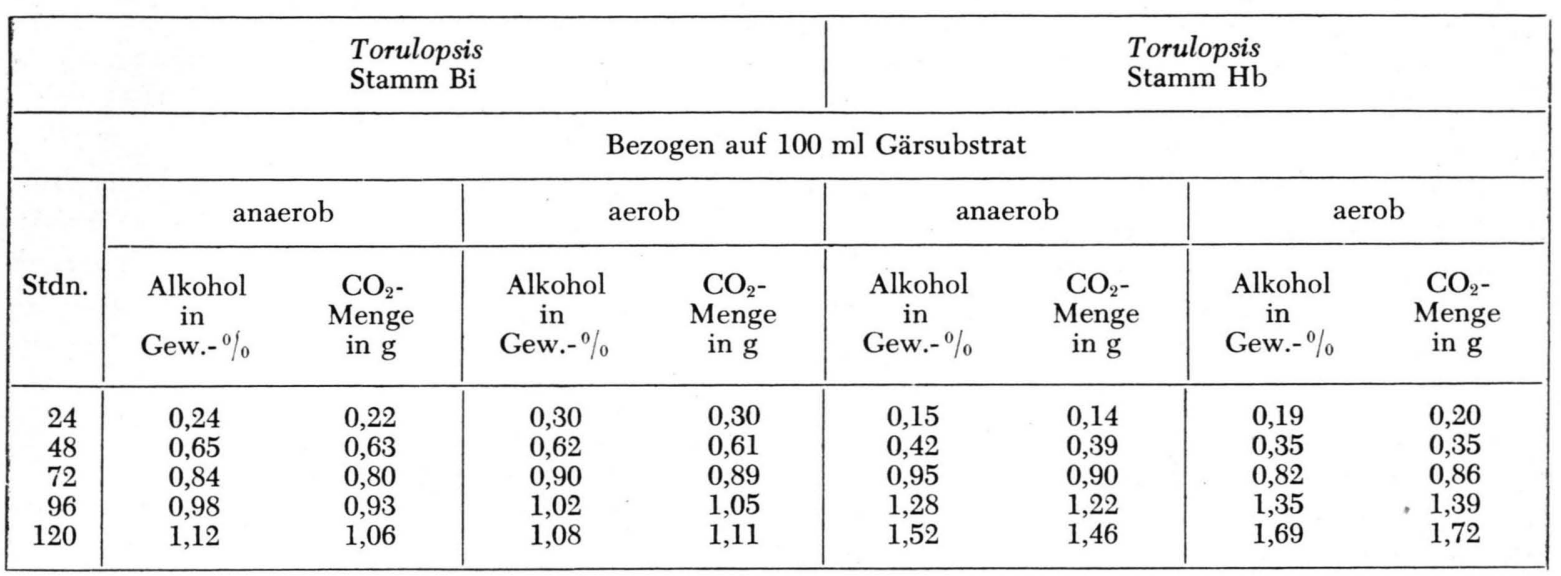

Tab. 4. Anaerobe und aerobe Alkoholbildung sowie $\mathrm{CO}_{2}$-Entwicklung der Torulazeen Stamm Bi und $\mathrm{Hb}$ in 12 -proz. Würze bei nicht-zuwachsfähiger Aussaatmenge. Raumtemperatur $10^{\circ}$.

einem größeren Einzelansatz zusammenzufassen, wobei wir wiederum genauestens die von $\mathrm{Meyerh}$ of eingehaltenen Versuchsbedingungen befolgten. Die hierbei erzielten Ergebnisse stimmten mit denen in der W a rburg-Apparatur erhaltenen gut überein und zeigten, daß die aerobe Alkoholbildung auch bei größerer Dimensionierung des Einzelversuches im gleichen Maße auftritt, wie es zuvor bei der Ansammlung einer Reihe von Kleingäransätzen der Fall war.

Die Bestätigung der annähernd gleichwertigen aeroben und anaeroben Alkoholbildung im Kleinund Großansatz mit Zuckerphosphatlösung veranlaßte uns, weitere Untersuchungen in Würze vorzunehmen, und zwar in Parallele zu den vorangehenden Testen an Weinhefen. Als Beispiel für den experimentellen Ablauf bringen wir im folgenden aus einer Gruppe von 11 Vertretern der Torulopsisfamilie, die übereinstimmende Resultate lieferten, die Versuchsdaten von Stamm Hb und Bi zur Kenntnis.

Ansatz 1: 10 Gärkolben mit je $100 \mathrm{ml}$ 12-proz. Würze $\left(p_{\mathrm{H}} 5,53\right)$; je Charge $1,5 \mathrm{~g}$ abgepreßte Torulopsishefe Stamm $\mathrm{Hb}$ (nicht-zuwachsfähige Aussaatmenge); Wassergehalt der Hefe: 79,7\%, Zellenzahl/ $/ \mathrm{mm}^{3}$ : 106500 ; anaerob: bei schwachem $\mathrm{N}_{2}$-Gasstrom (etwa 20 Gasblasen /min), aerob: bei schwachem $\mathrm{O}_{2}$-Gasstrom (etwa 20 Gasblasen/min). Raumtemperatur $10^{\circ}$.

M.St.-Bestimmungen:

$$
\begin{aligned}
& Q_{\mathrm{Al}}^{\mathrm{N}_{2}}=496 ; \quad Q_{\mathrm{Al}}^{\mathrm{O}_{2}}=500 ; \\
& Q_{\mathrm{CO}_{2}}^{\mathrm{N}_{2}}=240 ; \quad Q_{\mathrm{CO}_{2}}^{\mathrm{O}_{2}}=259 ; \quad G-Q_{\mathrm{CO}_{2}}^{\mathrm{O}_{2}}=242 ; \\
& Q_{\mathrm{O}_{2}}=-17 ; \quad A-Q_{\mathrm{O}_{2}}=-17 ; \quad \dot{U}-Q_{\mathrm{O}_{2}}=0 .
\end{aligned}
$$

Ansatz 2: 10 Gärkolben mit je $100 \mathrm{ml}$ 12-proz. Würze ( $p_{\mathrm{H}}$ 5,53); je Charge 1,5 g abgepreßte Torulopsishefe Stamm Bi (nicht-zuwachsfähige Aussaatmenge); Wassergehalt der Hefe: 80,3\%, Zellenzahl/ $\mathrm{mm}^{3}: 99200$; weitere Arbeitsbedingungen wie bei Ansatz 1. Raumtemperatur $10^{\circ}$.

M.St.-Bestimmungen:

$$
\begin{aligned}
& Q_{\mathrm{Al}}^{\mathrm{N}_{2}}=550 ; \quad Q_{\mathrm{Al}}^{\mathrm{O}_{2}}=556 ; \\
& Q_{\mathrm{CO}_{2}}^{\mathrm{N}_{2}}=266 ; \quad Q_{\mathrm{CO}_{2}}^{\mathrm{O}_{2}}=283 ; \quad G-Q_{\mathrm{CO}_{2}}^{\mathrm{O}_{2}}=269 ; \\
& Q_{\mathrm{O}_{2}}=-14 ; \quad A-Q_{\mathrm{O}_{2}}=-14 ; \quad \ddot{U}-Q_{\mathrm{O}_{2}}=0 .
\end{aligned}
$$

In der nachfolgenden Tab. 4 sind die Zahlenwerte der aeroben bzw. anaeroben Alkoholbildung und $\mathrm{CO}_{2}$-Entwicklung in 12-proz. Würze von $24 \mathrm{zu}$ $24 \mathrm{Stdn}$. innerhalb von 5 Gärtagen zusammengestellt.

Bei dem täglichen Verfolg der aeroben bzw. anaeroben Alkoholbildung und $\mathrm{CO}_{2}$-Entwicklung tritt unverkennbar zutage, daß die beiden zymagen umgezüchteten Torulopsishefen, Stamm $\mathrm{Hb}$ und Bi, ein spezifisches aerobes Gärvermögen besitzen; zwischen ihrer aeroben und anaeroben Stoffwechseltätigkeit macht sich kein hervortretender Unterschied geltend.

Die zuckerspaltende Torulopsis, von M e y e r h of als Prototyp der aerob nicht-gärenden Hefen herausgestellt, bildet oxy- wie anoxybiotisch annähernd in gleichem Ausmaß Alkohol. Das ist der elementare Befund unserer vorliegenden Untersuchungen im Warburg-Apparat sowie im entsprechend größer dimensionierten Zuckerphosphat- und Würze-Ansatz. Als entscheidendes Kriterium diente uns hierbei die Alkoholbestimmung, die - wie am Beispiel von Torulopsis besonders evident geworden - bei der 
quantitativen Differenzierung der beiden Stoffwechselarten nicht durch alleinige $\mathrm{CO}_{2}$-Messung ersetzt werden kann. In qualitativer Hinsicht eruierten wir jüngstens ${ }^{22}$, daß der Spaltungsstoffwechsel, zumindest was das Zellwachstum anbetrifft, ergonisch nicht für die Atmung einzutreten vermag.

Mit unserer Feststellung, daß die zuckerspaltende Torulopsis - in gleicher Weise wie die Kultur- und Weinhefen - einen normalen Gärungsstoffwechsel vollführt, stellen wir eine irrige Auffassung richtig, die seit M e y e rh of s unitarischer Korrelationstheorie die Zellstoffwechsellehre belastet. Das M e y e r h o f sche Experiment, aus dem hervorzugehen schien, daß die Torulopsishefe zu einer abnormen Atmungssteigerung fähig sei, blieb von vornherein fragwürdig, da ein unkoordinierter Zuckerabbau (typisch für die Gärung), der den Energiebedarf der Zelle um ein Vielfaches überschreitet, physiologisch dem Wesen der Atmung als zellular reguliertem Vorgang widerspricht, was aus der Berechnung der kalorischen Werte für Atmung und Gärung - bei einer totalen Umschaltung von $Q_{\mathrm{CO}_{2}}=250$ bis 300 auf Atmung, wie Meyerhof ${ }^{23}$ annimmt - leicht zu erkennen ist.

\section{Versuche mit Milchsäurebakterien}

Von 15 aus der Natur isolierten Stämmen, die bei hoher Aussaat aerob wie anaerob ein gleich gutes Säuerungsvermögen aufzuweisen hatten, sind nachfolgend die experimentell ermittelten Säuerungswerte von zwei typischen Vertretern, Bact. Delbrücki Leichmann und Bact. lactis acidi Leichmann, bei $\mathrm{O}_{2}-$ und $\mathrm{N}_{2}$-Durchgasung vergleichend gegenübergestellt.

Ansatz 1: 10 Gärkolben mit je $100 \mathrm{ml}$ 10-proz. Würze $\left(p_{\mathrm{H}} 5,60\right)$, unter Zusatz von 3 g sterilem $\mathrm{CaCO}_{3}$; je Charge 2,0 g lufttr. Milchsäurebakterien, Stamm Delbrücki Leichmann; Wassergehalt der Bakterien: 82,4\%; anaerob: bei schwachem $\mathrm{N}_{2}$-Gasstrom (etwa 20 Gasblasen/min), aerob: bei schwachem $\mathrm{O}_{2}$-Gasstrom (etwa $20 \mathrm{Gasblasen} / \mathrm{min}$ ). Thermostaten-Temperatur $42^{\circ}$.

Ansatz 2: 10 Gärkolben mit je $100 \mathrm{ml}$ 10-proz. Würze $\left(p_{\mathrm{H}} 5,60\right)$, unter Zusatz von 3 g sterilem $\mathrm{CaCO}_{3}$; je Charge $2,0 \mathrm{~g}$ lufttr. Milchsäurebakterien, Stamm lactis acidi Leichmann; Wassergehalt der Bakterien: 83,8\%; weitere Arbeitsbedingungen wie bei Ansatz 1. Thermostaten-Temperatur $38^{\circ}$.

In der nachstehenden Tab. 5 ist der Verlauf der Säurebildung unter aeroben und anaeroben Versuchsbedingungen, in Abständen von je 12 Stdn., bis zur Beendigung des Säuerungsprozesses vergleichend re-

$22 \mathrm{~F}$. Windisch, H. Ha ehn u. W. Heumann, Arch. Geschwulstforsch. (im Druck).

23 O. M e y e r h of, Biochem. Z. 162, 43, 59 [1925]. gistriert. Die quantitative Bestimmung der Milchsäure erfolgte in einem aliquoten Teil und wurde in jedem Falle auf $100 \mathrm{ml}$ Ausgangssubstrat umgerechnet.

Die aus der Natur isolierten und in hoher Aussaatmenge zum Versuch angesetzten beiden Bakterienstämme Delbrücki Leichmann und lactis acidi Leichmann ließen im aerob und anaerob durchgeführten Experiment keinen wesentlich sich auswirkenden Unterschied in der Intensität der Säurebildung erkennen. Das gleiche Ergebnis war auch bei

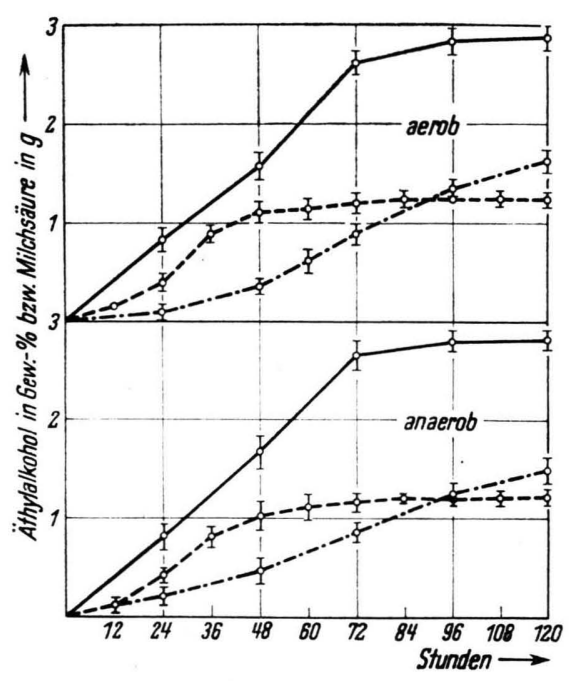

Abb. 2. Vergleichende aerobe und anaerobe Alkohol- bzw. Milchsäurebildung durch Weinhefe (Sacch. ellipsoideus Hansen) Stamm Rie (- Torulopsis Stamm Hb (-.----) und Bact. lactis acidi Leichmann (-----) bei nicht-zuwachsfähiger Aussaatmenge. - Die Kurven stellen die Resultierenden aus einer größeren Reihe von Versuchen dar. - bezeichnet die Streubereiche

den übrigen 13 Wildstämmen zu verzeichnen. Hieraus ist zu entnehmen, daß die Milchsäurebakterien, die aus der Luft stammen und spontane Säuerung hervorrufen, unabhängig vom Sauerstoff die glykolytische Spaltung des Zuckers vollführen.

Erläuterung zur vergleichenden graphischen Darstellung der Ergebnisse

In Abb. 2 sind die Durchschnittswerte einer größeren Reihe von Versuchen unter Angabe der Streubereiche graphisch dargestellt. Dabei mußten wir die analytischen Daten der aeroben und anaeroben Ansätze in getrennten Diagrammen bringen, weil sich andernfalls die Kurven bei ihrem ziemlich gleichartigen Verlauf im $\mathrm{O}_{2^{-}}$und $\mathrm{N}_{2}$-Durchgasungsversuch gegenseitig überdeckt hätten. 


\begin{tabular}{|c|c|c|c|c|}
\hline & \multicolumn{2}{|c|}{$\begin{array}{c}\text { Bact. Delbrücki } \\
\text { Leichmann }\end{array}$} & \multicolumn{2}{|c|}{$\begin{array}{c}\text { Bact. lactis acidi } \\
\text { Leichmann }\end{array}$} \\
\hline \multicolumn{5}{|c|}{ Bezogen auf $100 \mathrm{ml}$ Gärsubstrat } \\
\hline \multirow{2}{*}{ Stdn. } & \multicolumn{2}{|c|}{ Milchsäure in $\mathrm{g}$} & \multicolumn{2}{|c|}{ Milchsäure in $\mathrm{g}$} \\
\hline & anaerob & aerob & anaerob & aerob \\
\hline 12 & 0,26 & 0,19 & 0,11 & 0,15 \\
\hline 24 & 0,95 & 0,80 & 0,42 & 0,38 \\
\hline 36 & 1,48 & 1,54 & 0,85 & 0,92 \\
\hline 48 & 1,69 & 1,67 & 1,05 & 1,10 \\
\hline 60 & 1,79 & 1,82 & 1,11 & 1,17 \\
\hline 72 & 1,88 & 1,96 & 1,15 & 1,20 \\
\hline 84 & 1,92 & 1,97 & 1,21 & 1,24 \\
\hline 96 & 1,97 & 2,00 & 1,18 & 1,26 \\
\hline 108 & 1,95 & 1,97 & 1,15 & 1,26 \\
\hline 120 & 1,98 & 2,01 & 1,22 & 1,28 \\
\hline
\end{tabular}

Tab. 5. Anaerobe und aerobe Milchsäurebildung der Stämme Bact. Delbrücki Leichmann und Bact. lactis acidi Leichmann in 10-proz. Würze bei hoher Aussaatmenge. Züchtungstemperatur $42^{\circ}$ bzw. $38^{\circ}$.

\section{Dervegetative Einflußdes Sauerstoff} auf die Hefezelle

Eine Wechselwirkung von Atmung und Gärung, wie sie beim Warmblüter- und Kaltblütermuskel vorherrscht, besteht weder beim Krebsgewebe (W a rburg) noch bei den Kulturhefen (P a s teur, M e y e r h of); sie tritt ebensowenig - nach unseren vorliegenden Ergebnissen - bei frisch eingefangenen Wildhefen und Milchsäurebakterien zutage.

Die Folge des Lebens mit und ohne Luft wirkt sich in ganz anderem Sinne, viel tiefgreifender auf die einzelne Zelle aus, nämlich vegetativ, zellkonstitutionell, was in früheren Arbeiten ${ }^{24}$ von uns bereits herausgestellt wurde. Ähnliche Gedankengänge hatte auch schon Delbrück ${ }^{25}$ entwickelt: „Wenn von einem gärungsfördernden Einfluß der Lüftung die Rede ist, so will das in erster Linie heißen, daß durch die Luftzufuhr die Zahl der Zellen vermehrt und dadurch die Gesamtgärleistung erhöht wird. Gerade diese Eigenschaft der Luft, schon in geringen Mengen die Sproßtätigkeit der Hefe wesentlich zu erhöhen, verleiht ihr einen außerordentlichen Einfluß auf den Charakter der am Ende der Gärung ge-

24 F. W i n d i s c h, Hoppe-Seiler's Z. physiol. Chem. 179, 88 [1928]; Biochem. Z. 246, 332, $380 \mathrm{ff}$. [1932].

25 M. D e l b r ü c k u. F. H a y d u c k, Die Gärungsführung, Berlin 1911.

$26 \mathrm{~F}$. Windis ch, W. Heumann u. Chr. Gos li c h, Z. Naturforschg. 8 b, 305 [1953]; F. W i n d i s c h, H. Haehn u. W. Heumann, Arch. Geschwulstforsch. (im Druck). ernteten Hefe." Auch äußerte Delbrück (l.c. ${ }^{25}$, S. 29), daß die obergärigen Hefen sauerstoffbedürftiger seien als die untergärigen, weil sie „wüchsiger“ seien.

Tatsächlich gibt es für die Hefezelle, da sie im ausgereiften, ruhenden Zustand keiner $\mathrm{O}_{2}$-Beeinflussung unterliegt, nur ein rein vegetatives Moment der $\mathrm{O}_{2}$-Gebundenheit, einmal im Hinblick auf die Proliferation selbst ${ }^{26}$ und zweitens bezüglich der dabei sich entwickelnden Stoffwechselkräfte. Die potentielle Abhängigkeit der letzteren vom Sauerstoff läßt, aus der vegetativen Perspektive gesehen, die Pasteursche

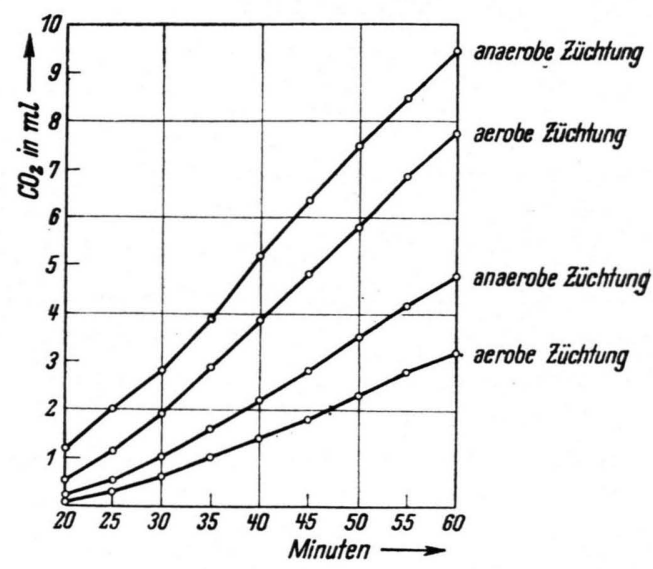

Abb. 3. Gärungsintensitäten von anaerob und unter Lüftung gezüchteten Hefen. Die beiden oberen Kurven (anaerobe und aerobe Züchtung) betreffen Kulturhefe Stamm $\mathbf{F}$, die beiden unteren Torulopsis Stamm Bi.

„conséquence de la vie sans air" voll in Erscheinung treten, was auch in der nachstehenden graphischen Darstellung der aerob und anaerob entwickelten Gärungsintensitäten (Abb. 3) mit aller Deutlichkeit zum Ausdruck kommt.

Anaerobe Züchtung (auch schon Lufthungerzüchtung) steigert die Gärkraft, aerobe vermindert sie, ohne sie vollständig auszulöschen; das gleiche trifft auch für die Atmungsintensität $\mathrm{zu}$, wie bereits früher von uns dargetan wurde ${ }^{27}$. Es findet also unter ananroben (auch schon unter $\mathrm{O}_{2}$-armen) Wachstumsbedingungen eine Potenzierung der beiderseitigen Stoffwechselkräfte statt, während aerob, besonders bei Belüftung, das Gegenteil eintritt. Ähnliches kommt in der Feststellung $\mathrm{Tr}$ a utweins ${ }^{28}$ zum Ausdruck, daß „Hefen, die eine große Gärungs-

27 F. W in dis c h, Ergebn. Enzymforsch. 2, 169 [1933].

$28 \mathrm{~K}$. Trautwein u. J. W as sermann, Biochem. Z. 229, 128 [1922]. 
geschwindigkeit haben, auch eine hohe Atmungsgeschwindigkeit besitzen“. Für die fertig ausgebildete, nicht in Vermehrung befindliche Hefezelle gibt es weder eine Pasteursche Konsequenz der Anaerobiose noch eine Meyerhofsche Korrelation zwischen Oxy- und Anoxybiose.

\section{Schlußbetrachtung}

Mit der Entdeckung der Krebsglykolyse, die gegenüber der aerob alternierenden Glykolyse der normalen Körperzellen in vivo - als aerob persistent determiniert ist, hat $\mathrm{W}$ a r b u r g gleichzeitig die bedeutsame Feststellung gemacht, daß es, zellfunktionell, zwei differente Arten von Spaltungsstoffwechsel gibt, die aerob reversible und die aerob irreversible (persistente). Auf Grund unserer vorliegenden experimentellen Ergebnisse erweitert und vertieft sich die W a r b u r g sche Erkenntnis dahingehend, daß außer den Krebszellen auch die Hefen und Milchsäurebakterien von Natur aus über die Fähigkeit der aeroben Zuckerspaltung verfügen. Demnach treten die beiden Prinzipien der Spaltwirkung nicht nur bei der malignen Entartung von Zellen divergent in Erscheinung, sondern machen sich ebenso im normalen Stoffwechselkreislauf (Hefen und Bakterien) geltend. Nach unserer Auffassung kommt hierbei dem aeroben Spaltungsstoffwechsel als unkoordiniert wirkender Naturkraft (P. Lindner hat die Hefe als „Zuckerzerstörungspilz" bezeichnet) die spezielle Aufgabe

29 F. W in dis ch, Naturwiss. 34, 190 [1947], zit. in B. F l a s chenträger, Physiologische Chemie, Berlin 1951, S. 1170; F. W in d is c h, Apoth.Ztg. 62, 86 [1949]. $\mathrm{zu}$, die kohlenhydrathaltigen (mineralische Bestandteile einschließenden) Ausscheidungs- und Abfallstoffe in der Natur, unabhängig von atmosphärischen $\mathrm{O}_{2}$-Einflüssen, so schnell wie möglich wieder assimilierbar, d. h. dem Aufbau zugänglich zu machen.

Die zymagene $\left(\mathrm{O}_{2}\right.$-arme) Umzüchtung der aus der Natur isolierten und ausschließlich atmenden Torulazeen zu aerob zuckerspaltenden Zellen, deren mutierter Stoffwechselmodus gegenüber jeglicher $\mathrm{O}_{2}$ Einwirkung irreversibel geworden ist, stellt gleichsam ein Paradigma zum Krebsgeschehen dar. Auch bei der Karzinogenese vollzieht sich nach der herrschenden zellularen Anschauung - primär infolge Sauerstoffmanges, wie $\mathrm{W}$ a r b u r g supponiert - die Umwandlung von ursprünglich aerob nur atmenden Normalzellen in malignes Gewebe, dessen ausgeprägter aerob glykolytischer Zellstoffwechsel für alle bösartigen Geschwülste symptomatisch ist. Die W a r burgsche Konzeption über die Ätiologie des Krebses fand ihre experimentelle Bestätigung, als es W in d i s ch ${ }^{29} 1947$ erstmalig in Vitrokultur gelang, normales tierisches Gewebe unter dem Einfluß der Anoxybiose in Krebsgewebe umzuzüchten. Letzthin konnten auch die amerikanischen Forscher Goldblatt und Cameron ${ }^{30}$ aus den medizinischen Instituten der Universität Los Angeles in großangelegten Versuchen unser anoxybiotisches Züchtungsergebnis bestätigen, welches im Sinne der Warburg s chen Intuitionen für die zellphysiologische Krebsforschung richtungweisend ist.

30 H. G o l d b l a t t u. C. C a m e r o n, J. exp. Medizine 97, 525 [1953]. 